\title{
World AIDS Day 2008
}

\section{NSMB observes World AIDS Day by reflecting on the crucial role of basic research in fighting this epidemic.}

$\mathbf{T}$ The first day of December marks World AIDS Day, established in 1988 to raise awareness about this disease. AIDS, or acquired immunodeficiency syndrome, corresponds to the later clinical stages of HIV infection. The numbers from the HIV/AIDS pandemic are overwhelming: 33 million people were living with HIV in 2007, with 2.5 million new infections and 2.1 million deaths. Worldwide, that translates to 6,800 people becoming infected and 5,700 dying from AIDS every single day.

Infection typically occurs through unprotected sexual intercourse or sharing of contaminated needles. Although so far there is no cure or vaccine for HIV/AIDS, transmission can be largely prevented by prophylaxis. For those infected, there are antiretroviral drugs that can keep the viral titers under control and decrease the morbidity and mortality associated with HIV infection. But poverty, lack of information and the outcast status of some high-risk groups (such as sex workers and injecting drug users) have been barriers to intervention in several countries. In addition, antiretroviral drugs can be expensive and have considerable side effects, making patient compliance an important concern, particularly because the emergence of resistant variants increases when the drug regimen is not followed properly, exacerbating the need for novel drugs.

Our current knowledge of HIV/AIDS, which guides public health initiatives to fight the epidemic, and the very existence of a therapy are the fruits of intense research from scientists in different fields and settings, including the pharmaceutical industry. A PubMed search with the words "HIV" and "AIDS" finds over 80,000 papers since the syndrome was described in the early 80 s, followed by the pivotal identification of its causative agent, a discovery whose importance was recognized this year by the Nobel Prize in Physiology or Medicine awarded to Françoise Barré-Sinoussi and Luc Montagnier. The discovery of the virus allowed the development of diagnostic tools, essential for avoiding infection through blood transfusions and other medical procedures, and for identifying patients in the early phases of infection, who do not show symptoms but can nevertheless transmit the virus. Epidemiological studies have allowed us to understand the patterns of HIV spread and how the infected population has changed, with an increasing proportion of women being affected worldwide, which has important consequences, as the virus can be transmitted from mother to child during birth and by breastfeeding.

The pathogenesis and the viral life cycle of HIV and closely related retroviruses have been and continue to be actively studied, and each event or interaction is a potential target for therapeutic intervention. Among the drugs used at present are inhibitors of the viral reverse transcriptase and protease; more recent drugs target integrase and the gp 41 region of the viral envelope to inhibit the fusion process required for viral entry. Information about how the virus exploits the cellular machinery for its own replication, as well as the tug-of-war between the virus and cellular defenses, has emerged from intensive basic research, some of which has been published in our pages. For example, two papers described the interactions between Alix and viral protein p6, which are essential for viral budding. The interplay between viral protein Vif and host defense enzyme APOBEC $3 \mathrm{G}$ has also been recently described. In this issue (p. 1287), Geyer and colleagues examine the complex of cyclin T1 with the related EIAV protein Tat and RNA element TAR, which activates viral transcription. Such detailed knowledge is crucial for understanding HIV biology and guiding rational therapeutic design.

The field was recently excited by reports of an HIV-infected patient effectively cured by a bone marrow transplant. This patient had to undergo a bone marrow transplant to treat leukemia and, after 20 months without antiretroviral therapy, has no detectable virus in circulation. His doctors had used bone marrow from a donor who was homozygous for a deletion mutation in the gene encoding a co-receptor for HIV, CCR5, that is required for viral entry into host cells. This particular CCR5 gene mutation, called $\Delta 32$, is common among the northern European population (10\% frequency), and homozygosity results in loss of CCR5 cell surface expression. The mutation was identified in 1997 from samples of individuals who had been exposed to HIV multiple times but remained uninfected. There is no apparent disadvantage in the absence of CCR5, perhaps because of redundancy among chemokine receptors, and there is discussion of whether this mutation was previously subject to positive selection.

The news about this putative cure is certainly intriguing, but bone marrow transplant cannot be used as a standard therapy for HIV. It is a harsh treatment, wherein the patient's own immune system cells are destroyed by chemotherapy and radiation and then replaced by bone marrow material from a compatible donor; $10-30 \%$ of patients do not survive. But it does point to the feasibility of using RNA interference to suppress CCR5 expression in HIV patients, as has been shown in cell cultures and in rhesus macaques. However, HIV-1 variants that use CXCR4 as co-receptor instead of CCR 5 appear in $~ 50 \%$ of patients at later stages of the disease, and it is not yet known why this transition occurs.

The holy grail of HIV/AIDS research remains unarguably the development of an effective vaccine. The results from clinical trials have been disappointing, to say the least. A major obstacle for HIV therapy in general is the rapid adaptability of HIV, a result of the high rate of mutation during the reverse transcription reaction. The focus has shifted to protein regions that are less prone to mutations due to functional constraints, such as those involved in fusion, which could be a target for a therapeutic vaccine. Another essential caveat is that we still do not know what constitutes protective immunity. The failures in vaccine development have prompted experts in the field to point to the need for more basic science and interdisciplinary approaches, with calls for funding of more creative and innovative research (which also translates to high-risk projects). This might be difficult to do in times of shrinking research budgets, but it is a necessary investment if we want to be able to respond to the global disaster of HIV/AIDS. 